ELECTRONIC RESEARCH ANNOUNCEMENTS

OF THE AMERICAN MATHEMATICAL SOCIETY

Volume 6, Pages 52-63 (July 19, 2000)

S $1079-6762(00) 00081-0$

\title{
ON THE CONNECTEDNESS OF THE SPACE OF INITIAL DATA FOR THE EINSTEIN EQUATIONS
}

\author{
BRIAN SMITH AND GILBERT WEINSTEIN
}

(Communicated by Richard Schoen)

\begin{abstract}
Is the space of initial data for the Einstein vacuum equations connected? As a partial answer to this question, we prove the following result: Let $\mathcal{M}$ be the space of asymptotically flat metrics of non-negative scalar curvature on $\mathbb{R}^{3}$ which admit a global foliation outside a point by 2 -spheres of positive mean and Gauss curvatures. Then $\mathcal{M}$ is connected.
\end{abstract}

\section{INTRODUCTION}

The Einstein vacuum equations of general relativity read:

$$
\bar{R}_{\mu \nu}-\frac{1}{2} \bar{R} \bar{g}_{\mu \nu}=0,
$$

where $\bar{R}_{\mu \nu}$ is the Ricci curvature tensor of a Lorentzian 4-manifold, and $\bar{R}$ the scalar curvature. The basic problem for these equations is the Cauchy problem: given data on a time-slice $M$, consisting of a Riemannian metric $g$ and a second fundamental form $k$ on $M$, find the evolution of space-time according to (11). Not all the equations in (11) are evolution equations. Using the twice-contracted Gauss equation and the Codazzi equations of the Riemannian submanifold $M$, one finds that the normal-normal and normal-tangential components of (1) are:

$$
\begin{aligned}
R-|k|^{2}+(\operatorname{tr} k)^{2} & =0, \\
\nabla^{j} k_{i j}-\nabla_{i} \operatorname{tr} k & =0,
\end{aligned}
$$

where $R$ is the scalar curvature of $M$, and $k$ its second fundamental form. These equations, called the Vacuum Constraint Equations, involve no time derivatives and hence are to be considered as restrictions on the data $g$ and $k$; see [14. We will only consider asymptotically flat (AF) solutions of these equations, i.e., solutions satisfying the decay:

$$
\begin{aligned}
g_{i j}-\delta_{i j} & =O\left(r^{-1}\right), \\
k_{i j} & =O\left(r^{-2}\right), \\
R & \in L^{1}(M) .
\end{aligned}
$$

Received by the editors May 27, 1999.

2000 Mathematics Subject Classification. Primary 83C05; Secondary 58G11.

This research was supported in part by NSF grant DMS 9704760.

(C)2000 American Mathematical Society 
It is standard to choose the maximal gauge tr $k=0$ in (2)-(3), which, as shown by Bartnik [1], involves no loss of generality. In this case, we get the Maximal Gauge Vacuum Constraint Equations:

$$
\begin{aligned}
R & =|k|^{2}, \\
\operatorname{tr} k & =0, \\
\operatorname{div} k & =0 .
\end{aligned}
$$

These form an underdetermined system of elliptic equations on $M$ for $g$ and $k$.

Much work has been devoted to finding solutions of (4)-(6); see for example [3, 4. 6] 7, 9, 10, 15, and the references therein. However, certain fundamental questions remain unanswered. For example, it is not known whether the space of AF initial data on a given 3-manifold $M$ is connected, not even in the case $M=\mathbb{R}^{3}$. Since the evolution equations trace a continuous path in the phase space of initial data, either answer to this question would be of considerable significance for the dynamics of the Einstein equations.

The standard method for solving (44)-(6) has been the conformal method. In this method the free data is the conformal class of an asymptotically flat Riemannian metric $g$, and a trace-free divergence-free symmetric 2-tensor $k$ on $M$. Since the trace-free and divergence-free conditions on $k$ are invariant under the transformation $g \mapsto \phi^{4} g, k \mapsto \phi^{-2} k$, it suffices to find $\phi$ so that (4) is satisfied. This will be so provided that the Lichnerowicz equation is satisfied:

$$
\Delta \phi-\frac{1}{8} R \phi+|k|^{2} \phi^{-7}=0 .
$$

A solution of this equation can be found if the negative part of the scalar curvature is small enough in the $L^{3 / 2}$ norm; see [9. In particular, the question above can be reduced to the following purely geometric problem: is the space of AF metrics of non-negative scalar curvature on a 3 -manifold $M$ connected?

In this paper, we announce, and sketch the proof of a result which gives a partial answer in the affirmative to the question posed above; details will appear in [13]. We say that a topological 2-sphere $S$ in $M$ is quasiconvex if both the Gauss and the mean curvature of $S$ are positive [8]. Let $\mathcal{M}$ be the space of $C_{-1}^{2, \alpha}$ metrics $g$ on $\mathbb{R}^{3}$ with non-negative scalar curvature $R \in L^{1}$ which admit a global coordinate system whose coordinate spheres are quasiconvex, and which satisfy in this coordinate system:

$$
\begin{array}{ll}
g_{i j}-\delta_{i j}=O\left(r^{-1}\right) & \text { as } r \rightarrow \infty, \\
g_{i j}-\delta_{i j}=0 & \text { at } r=0 .
\end{array}
$$

The $C_{-1}^{2, \alpha}$ topology on $\mathcal{M}$ is generated by the following system of neighborhoods of any metric $g \in \mathcal{N}$ :

$$
\left\{g^{\prime} \in \mathcal{M}: \sum_{\alpha=0}^{2} \sup \left|(1+r)^{m+1} \partial^{m}\left(g_{i j}-g_{i j}^{\prime}\right)\right|+\left[\partial^{2} g_{i j}-\partial^{2} g_{i j}^{\prime}\right]_{\alpha,-3}<\epsilon\right\},
$$

where

$$
[f]_{\alpha,-k}=\sup _{r}\left((1+r)^{k+\alpha} \sup _{x, y \in B_{r}} \frac{|f(x)-f(y)|}{|x-y|^{\alpha}}\right)
$$


is the weighted Hölder norm with exponent $\alpha$ of $f$ on $\mathbb{R}^{3}$. In fact, in view of the general covariance of the Einstein Equations, we are only interested in the quotient of $\mathcal{M}$ by the group $\mathcal{G}$ of diffeomorphisms of $\mathbb{R}^{3}$.

Main Theorem. The quotient of $\mathcal{M} / \mathcal{G}$ is path connected in the quotient topology induced by $C_{-1}^{2, \alpha}$ on $\mathcal{M}$.

Of course, this raises the following question: when does an AF metric $g$ of nonnegative scalar curvature belong to $\mathcal{M}$ ? Clearly, a necessary condition is that $g$ possesses no compact minimal surfaces. However we do not even know whether the absence of compact minimal surfaces suffices to guarantee the existence of a global foliation with positive mean curvature.

To prove our Main Theorem, we generalize a method introduced by Bartnik [2] to construct quasispherical metrics of prescribed scalar curvature. A metric is quasispherical if it can be foliated by round spheres, spheres of constant curvature. Bartnik observed that prescribing scalar curvature for this type of metric could be viewed as a parabolic equation on the sphere for one of the metric coefficients, $u=|\nabla r|^{-1}$, where $r$ is the foliating function, provided that the mean curvature was also positive. We combine this with the Poincaré Uniformization as in 8 to get a general method to prescribe scalar curvature for metrics in $\mathcal{M}$. As an application, we prove the Main Theorem.

Denote by $r$ the foliating function normalized so that the area of the spheres is $4 \pi r^{2}$, and by $\gamma$ the induced metric on the spheres. Any smooth enough metric $g \in \mathcal{M}$ can be written as:

$$
g=u^{2} d r^{2}+e^{2 v} \bar{\gamma}_{A B}\left(\hat{\beta}^{A} d r+r d \theta^{A}\right)\left(\hat{\beta}^{B} d r+r d \theta^{B}\right),
$$

where $\left(\theta^{1}, \theta^{2}\right)$ are local coordinates on $\mathbb{S}^{2}, \bar{\gamma}_{A B}$ is a fixed (independent of $r$ ) round metric of area $4 \pi$, and $\hat{\beta}=\hat{\beta}^{A} \partial_{A}$ is the shift vector. Here, and throughout, we use the summation convention: repeated indices are summed over their range, $0,1,2,3$ for Greek indices, 1,2,3 for lower case Latin indices, and 1,2 for upper case Latin indices. Let $\chi$ be the second fundamental form, $H=\operatorname{tr}_{\gamma} \chi$ be the mean curvature of the spheres, and $\Pi=\mathcal{L}_{\hat{\beta}} \gamma$ be the deformation tensor of $\hat{\beta}$ on the spheres; then it can be checked that

$$
\begin{aligned}
\bar{\chi}=r u \chi & =\left(\left(1+r v_{r}\right) \gamma-\Pi / 2\right), \\
\bar{H}=r u H & =\left(2+2 r v_{r}-e^{-2 v} \operatorname{div}_{\bar{\gamma}} \beta\right),
\end{aligned}
$$

where $\beta=e^{2 v} \hat{\beta}$. It is important to note that both $|\bar{\chi}|_{\gamma}^{2}$ and $\bar{H}$ can be calculated in terms of only $\beta, v, r$, and the round metric $\bar{\gamma}$ on $\mathbb{S}^{2}$. Let $N$ be the outer unit normal to the foliation spheres, let $\bar{N}=r u N=r \partial_{r}-\hat{\beta}$, let $\phi_{\gamma}$ be the Laplacian on the spheres with respect to $\gamma$, and let

$$
\kappa=r^{-2} e^{-2 v}(1-\not \Delta v)
$$

be the Gauss curvature of the spheres. Then the equation for the scalar curvature $R$ of $g$ can be written as

$$
\bar{H} \partial_{\bar{N}} u=r^{2} u^{2} \phi_{\gamma} u+\bar{A} u-\bar{B} u^{3},
$$


where

$$
\begin{gathered}
\bar{A}=\partial_{\bar{N}} \bar{H}-\bar{H}+\frac{1}{2}|\bar{\chi}|_{\gamma}^{2}+\frac{1}{2} \bar{H}^{2}, \\
\bar{B}=r^{2}\left(\kappa-\frac{1}{2} R\right)=e^{-2 v}(1-\not \downarrow v)-\frac{1}{2} r^{2} R .
\end{gathered}
$$

Noting that the Laplacian with respect to $\bar{\gamma}$ is $\not{\alpha}=r^{2} e^{2 v} \boldsymbol{\phi}_{\gamma}$, we obtain, provided that $H>0$, the following Bernoulli-type parabolic equation for $u$ on the unit sphere:

$$
r \partial_{r} u-\beta \cdot \not \supset u=\Gamma u^{2} \not \Delta u+A u-B u^{3},
$$

where $\not \backslash u$ is the tangential component of the gradient of $u, \Gamma=e^{-2 v} / \bar{H}, A=\bar{A} / \bar{H}$ and $B=\bar{B} / \bar{H}$. It follows from the comment following equations (8)-(9) that the coefficients $\Gamma, A$ and $B$ can be calculated in terms of only $\beta, v, r$, the round metric $\bar{\gamma}$ on $\mathbb{S}^{2}$, and $R$. The quasispherical case can be recovered by setting $v=0$, and $\kappa=1$, see [2].

The proof of the Main Theorem is based on the study of equation (12). The deformation to a flat metric is accomplished in several steps. First, the metric is smoothed out with the scalar curvature $R$ truncated to be compactly supported. Next, we deform the metric to one satisfying $2 \kappa>R$. Then, we deform to a metric with compactly supported $\beta$ and $v$. Finally, we deform to a flat metric. The last three steps are all based on the following strategy. The deformation $g_{\lambda}$ is defined explicitly on a ball $B_{r_{0}}$. In the exterior of $B_{r_{0}}$ we consider $\beta_{\lambda}, v_{\lambda}$, and $R_{\lambda}$ as free data, and solve equation (12) on $\left[r_{0}, \infty\right) \times \mathbb{S}^{2}$ for $u_{\lambda}$ with initial conditions $\left.u_{\lambda}\right|_{S_{r_{0}}}$. In order for this to be feasible, and for the resulting metric $g_{\lambda}$ to yield a continuous path, we must ensure that $\beta_{\lambda}, v_{\lambda}$, and $R_{\lambda}$ are continuous in the appropriate spaces, that $\bar{H}_{\lambda}$ is positive, and that $R_{\lambda}$ is non-negative. In addition, one must verify conditions that guarantee the global existence of the solution $u_{\lambda}$, its appropriate decay as $r \rightarrow \infty$, and continuity with respect to $\lambda$. The regularity of $u_{\lambda}$ across $S_{r_{0}}$ is obtained by solving equation (12) on $\left[r^{\prime}, r_{0}+\epsilon\right) \times \mathbb{S}^{2}, 0<r^{\prime}<r_{0}$, with initial data $\left.u_{\lambda}\right|_{S_{r^{\prime}}}$ and by using the uniqueness and regularity of solutions.

The plan of the paper is as follows. In the next section, we derive equation (III). In Section 2, we collect the analytical results we need on existence, uniqueness, asymptotic behavior, and continuous dependence on parameters of solutions of (12). Then in Section 3, we sketch the proof of the Main Theorem by deforming any metric in $\mathcal{M}$ to the Euclidean metric.

\section{The SCALAR CURVATURE OF A 3-MANifold FOLIATED By SPHERES}

In this section, we derive equation (11). First, note that $\nabla r=u^{-1} N$, and consequently $u^{-1}=N^{i} \nabla_{i} r$. It follows that

$$
\nabla_{N} N=-u^{-1} \not \mathbf{u},
$$

where $\not \backslash u$ is the tangential part of $\nabla u$. Furthermore, using $N^{j} \nabla_{i} N_{j}=0$, we get

$$
\begin{aligned}
\nabla_{i} \nabla_{j} u^{-1} & =N^{k} \nabla_{i} \nabla_{j} \nabla_{k} r+\left(\nabla_{j} \nabla_{k} r\right)\left(\nabla_{i} N^{k}\right) \\
& =N^{k} \nabla_{i} \nabla_{k} \nabla_{j} r+\nabla_{j}\left(u^{-1} N_{k}\right)\left(\nabla_{i} N^{k}\right) \\
& =N^{k} \nabla_{k} \nabla_{i} \nabla_{j} r+u^{-1} R_{j l i k} N^{k} N^{l}+u^{-1}\left(\nabla_{j} N_{k}\right)\left(\nabla_{i} N^{k}\right),
\end{aligned}
$$


which after tracing with respect to $g$ leads to

$$
\Delta u^{-1}=\nabla_{N} \Delta r+u^{-1} \operatorname{Ric}(N, N)+u^{-1}|\nabla N|^{2},
$$

where Ric is the Ricci tensor of $g$. We now need to calculate the first and third terms on the right hand side of (14). Since the tangential part of $\nabla N$ is the second fundamental form $\chi$, and the normal part is given by (13), it is easy to see that:

$$
|\nabla N|^{2}=|\chi|_{\gamma}^{2}+u^{-2}|\not \chi u|_{\gamma}^{2} \text {. }
$$

Next, using equation (13) again, we find:

$$
\begin{aligned}
\nabla_{N} \Delta r & =N^{k} N^{i} \nabla_{k} \nabla_{i} u^{-1}+\left(\nabla_{N} N^{i}\right)\left(\nabla_{i} u^{-1}\right)+\nabla_{N}\left(u^{-1} \nabla^{i} N_{i}\right) \\
& =\nabla^{2} u^{-1}(N, N)+\left.u^{-3}|\not| u\right|_{\gamma} ^{2}+\nabla_{N}\left(u^{-1} H\right) .
\end{aligned}
$$

Substituting the last two equations into (14) gives

$$
\Delta u^{-1}=\nabla^{2} u^{-1}(N, N)+\left.2 u^{-3}|\not| u\right|_{\gamma} ^{2}+u^{-1} \operatorname{Ric}(N, N)+\nabla_{N}\left(u^{-1} H\right)+u^{-1}|\chi|_{\gamma}^{2},
$$

where $\nabla^{2} u^{-1}$ denotes the Hessian of the function $u^{-1}$. On the other hand:

$$
\Delta u^{-1}=\nabla^{2} u^{-1}(N, N)+H \nabla_{N} u^{-1}+\not \phi_{\gamma} u^{-1} .
$$

Combining the last two equations we obtain:

$$
\boldsymbol{\phi}_{\gamma} u=\left.2 u^{-1}|\not\rangle\right|^{2}-u^{2} \not u^{-1}=-u \operatorname{Ric}(N, N)-u \nabla_{N} H-u|\chi|^{2} .
$$

Substituting $\operatorname{Ric}(N, N)=\frac{1}{2}\left(H^{2}-|\chi|^{2}+R-\not R\right)$ from the Gauss equation, and the definitions $\bar{H}=r u H, \bar{\chi}=r u \chi$, and $\bar{N}=r u N$ from the introduction, we get (11).

\section{Bernoulli-type parabolic PDEs on $\mathbb{S}^{2}$}

In this section we collect the analytical results we need to prove the Main Theorem: (conditions for) global existence, uniqueness, asymptotic behavior and continuous dependence on parameters for solutions of (12). All these rely on a simple pointwise a priori bound whose proof we present here. Many of the results presented in this section are adapted from [2]. In order to ensure the uniform parabolicity of (12) we assume throughout this section that $\Gamma=e^{-2 v} / \bar{H}$ is bounded above and below by positive constants.

First, we define parabolic Banach spaces to be used in our study of (12). Let $0<r_{0}<r_{1} \leq \infty, I=\left[r_{0}, r_{1}\right] \subset \mathbb{R}^{+}$, and let $A_{I}=I \times \mathbb{S}^{2}$. Given a function $f$ on $A_{I}$, define:

$$
\begin{gathered}
{[f]_{\alpha ; I}=\sup _{\substack{\left(r_{1}, \theta_{1}\right),\left(r_{2}, \theta_{2}\right) \in A_{I} \\
\operatorname{dist}\left(\theta_{1}, \theta_{2}\right)<\pi}}\left[\frac{\left|f\left(r_{2}, \theta_{2}\right)-f\left(r_{1}, \theta_{1}\right)\right|}{\left|1-r_{2} / r_{1}\right|^{\alpha / 2}+\operatorname{dist}\left(\theta_{2}, \theta_{1}\right)^{\alpha}}\right],} \\
\|f\|_{0 ; I}=\sup _{A_{I}}|f|, \quad\|f\|_{0, \alpha ; I}=\|f\|_{0 ; I}+[f]_{\alpha ; I} .
\end{gathered}
$$

Here $\operatorname{dist}(\cdot, \cdot)$ denotes the geodesic distance on $\mathbb{S}^{2}$. If $f(r, \cdot)$ is a tensor field on $\mathbb{S}^{2}$, then $f\left(r_{2}, \theta_{2}\right)$ is understood to mean the parallel translate of $f\left(r_{2}, \theta_{2}\right)$ back to $\theta_{1}$ along the unique geodesic from $\theta_{1}$ to $\theta_{2}$. With these conventions, we can now define:

$$
\|f\|_{k, \alpha ; I}=\sum_{i+2 j \leq k}\left\|\not \not^{i}\left(r \partial_{r}\right)^{j} f\right\|_{0 ; I}+\sum_{i+2 j=k}\left[\not^{i}\left(r \partial_{r}\right)^{j} f\right]_{\alpha ; I}
$$


where $\not^{i} f$ is to be interpreted as the $i$-th covariant derivative of $f$ in the standard metric on $\mathbb{S}^{2}$. Now define $H_{I}^{k, \alpha}$ to be the space of functions $f$ on $A_{I}$ for which $\|f\|_{k, \alpha ; I}$ is defined and finite. Equipped with the norm $\|\cdot\|_{k, \alpha ; I}$, the space $H_{I}^{k, \alpha}$ is a Banach space. Following Bartnik in [2], we also use the notation:

$$
f^{*}(r)=\sup _{\theta \in \mathbb{S}^{2}} f(r, \theta), \quad f_{*}(r)=\inf _{\theta \in \mathbb{S}^{2}} f(r, \theta) .
$$

2.1. Conditions for global existence and uniqueness. Our first observation is that equation (12) is uniformly parabolic with $r$ as the 'time' variable. Therefore, given any initial data $u\left(r_{0}, \theta\right)=u_{0}(\theta)$, it is standard to obtain the existence of a unique solution on a short time interval $\left[r_{0}, r_{0}+\epsilon\right)$ for some $\epsilon>0$. Furthermore, it is well known that, for some choices of coefficients and initial data, a classical solution can blow up in finite time. Thus, our main objective here is to derive conditions which guarantee the existence of a global positive solution on the time interval $\left[r_{0}, \infty\right]$.

The principal ingredient in this and future subsections is a simple a priori bound on solutions of (12). To derive this bound, we use the familiar substitution $w=u^{-2}$ well-known from the elementary method used to solve the corresponding Bernoulli ordinary differential equation. If $u>0$ satisfies (12) on $\left[r_{0}, r_{1}\right]$, then $w$ satisfies

$$
r \partial_{r} w-\beta \cdot \not \downarrow u=2\left(-\Gamma u^{-1} \not \mathbf{\Delta} u-A w+B\right) .
$$

Since this equation is only used to derive pointwise a priori bounds, and since $u$ has a maximum where $w$ has a minimum and vice versa, there is no need to transform the gradient and Laplacian terms. For example, it follows from (15) that

$$
r \partial_{r} w_{*}+2 A^{*} w_{*} \geq 2 B_{*},
$$

which upon integration yields the lower bound:

$$
r w_{*} \geq\left[r_{0} w_{*}\left(r_{0}\right)+\int_{r_{0}}^{r} 2 B_{*} \exp \left(\int_{r_{0}}^{r^{\prime}}\left(2 A^{*}-1\right) \frac{d t}{t}\right) d r^{\prime}\right] \exp \left(\int_{r_{0}}^{r}\left(1-2 A^{*}\right) \frac{d t}{t}\right) .
$$

Similarly, one obtains the upper bound:

$$
r w^{*} \leq\left[r_{0} w^{*}\left(r_{0}\right)+\int_{r_{0}}^{r} 2 B^{*} \exp \left(\int_{r_{0}}^{r^{\prime}}\left(2 A_{*}-1\right) \frac{d t}{t}\right) d r^{\prime}\right] \exp \left(\int_{r_{0}}^{r}\left(1-2 A_{*}\right) \frac{d t}{t}\right) .
$$

In particular it follows immediately that $w$ is bounded above, and hence $u \geq c>0$, where $c$ depends on $r_{1}$. Suppose furthermore that

$$
K=\frac{1}{r_{0}}\left(\sup _{r_{0}<r<\infty}\left(-\int_{r_{0}}^{r} 2 B_{*} \exp \left(\int_{r_{0}}^{s}\left(2 A^{*}-1\right) \frac{d t}{t}\right) d s\right)\right)^{-1 / 2}>0 .
$$

If the initial data $u_{0}<K$, then $w$ is bounded below, and hence $u<\infty$. We note that if $B \geq 0$ then $K=\infty$, which gives $u<\infty$ for any positive initial data. The above considerations lead to the following result:

Theorem 1. Let $I=\left[r_{0}, \infty\right)$, suppose $\beta, \Gamma, A, B \in C^{\alpha}\left(I \times \mathbb{S}^{2}\right)$, and suppose that the constant $K$ defined in (19) is positive. Then, for any positive function $u_{0} \in C\left(\mathbb{S}^{2}\right)$ satisfying $\sup _{\mathbb{S}^{2}} u_{0}<K$, the equation (12) has a unique positive global classical solution $u$ on $I \times \mathbb{S}^{2}$ with initial data $u\left(r_{0}, \theta\right)=u_{0}(\theta)$. 
Now, suppose that the coefficients in (12) satisfy

$$
\|\beta\|_{2, \alpha ; I^{\prime}}+\|\Gamma\|_{2, \alpha ; I^{\prime}}+\|A\|_{2, \alpha ; I^{\prime}}+\|B\|_{2, \alpha ; I^{\prime}} \leq C,
$$

for some $C>0$, and that $u$ is a solution which is bounded above and below, $C^{-1} \leq u \leq C$ on $A_{I}$, where $I \subset I^{\prime}$. Then standard parabolic Schauder theory gives

$$
\|u\|_{4, \alpha ; I} \leq C^{\prime},
$$

where $C^{\prime}$ depends on $C$ and the length of $I$. Using the scaling properties of the $H_{I}^{k, \alpha}$-norms and of equation (12) we can also derive (20) for $I_{\lambda}=\left[\lambda r_{0}, \lambda r_{1}\right]$ with $C^{\prime}$ independent of $\lambda$ provided $I_{\lambda} \subset I^{\prime}$. Furthermore, if in addition $|2 A-1|<C / r$ on $\left[r_{0}, \infty\right) \times \mathbb{S}^{2}$ and $u\left(r_{0}, \theta\right)<K$, we may use the bounds (17) and (18) to obtain uniform infimum and supremum bounds on every subinterval of $\left[r_{0}, \infty\right)$. Thus, we obtain the following result.

Theorem 2. Let $I=\left[r_{0}, \infty\right)$, and suppose that there is a constant $C>0$ such that $\beta, \Gamma, A, B \in H_{I}^{2, \alpha}$ satisfy

$$
\begin{gathered}
\|\beta\|_{2, \alpha ; I},\|\Gamma\|_{2, \alpha ; I},\|B\|_{2, \alpha ; I},\|r(2 A-1)\|_{2, \alpha ; I} \leq C, \\
C^{-1} \leq \Gamma \leq C,
\end{gathered}
$$

and $u_{0} \in C^{4, \alpha}\left(\mathbb{S}^{2}\right)$ satisfies $0<u_{0} \leq K-C^{-1}$. Then there is a unique positive solution $u \in H_{I}^{4, \alpha}$ of (12) with initial condition $u\left(r_{0}, \theta\right)=u_{0}(\theta)$. Furthermore, we have

$$
\|u\|_{4, \alpha ; I} \leq C^{\prime}
$$

where $C^{\prime}$ depends only on $C$.

Our final result concerning global existence is a simple consequence of the maximum principle.

Theorem 3. Let $I=\left[r_{0}, \infty\right)$, and suppose that $u>0$ is a classical solution of equation (12) on $I \times \mathbb{S}^{2}$ with coefficients $\beta, \Gamma, A, B \in H_{I}^{2, \alpha}$ and with initial data $u_{0} \in C^{4, \alpha}\left(\mathbb{S}^{2}\right)$. Let $\tilde{B} \in H_{I}^{2, \alpha}$ satisfy $\tilde{B} \geq B$. Then the equation

$$
r \partial_{r} u-\beta \cdot \not 內 u=\Gamma u^{2} \not \Delta u+A u-\tilde{B} u^{3}
$$

has a unique solution $\tilde{u} \in H_{I}^{4, \alpha}$ with the same initial data $\tilde{u}\left(r_{0}, \theta\right)=u_{0}(\theta)$. Furthermore, we have $0<\tilde{u} \leq u$.

Proof. It suffices to prove a supremum a priori bound for $\tilde{u}$ on any interval $\left[r_{0}, r_{1}\right)$ where the solution $\tilde{u}>0$ exists. Subtracting the equation for $u$ from the equation for $\tilde{u}$, we get an equation for $v=\tilde{u}-u$ :

$$
r \partial_{r} v-\beta \cdot \not \downarrow v=\Gamma \tilde{u}^{2} \not \Delta v+\tilde{A} v-(\tilde{B}-B) \tilde{u}^{3},
$$

where $\tilde{A}=A+\Gamma(\tilde{u}+u) \not \mathbf{x} u-B\left(\tilde{u}^{2}+\tilde{u} u+\tilde{u}^{2}\right)$. Since $(\tilde{B}-B) \tilde{u}^{3}>0$, the maximum principle applies to give $v \leq 0$. It follows that $\tilde{u} \leq u$ on $\left[r_{0}, r_{1}\right)$.

For the remainder of Section 2 we set $I=\left[r_{0}, \infty\right)$. 
2.2. Asymptotic behavior. To study the asymptotic behavior of solutions of equation (12) we define:

$$
m=\frac{r}{2}\left(1-u^{-2}\right) .
$$

If $u$ is a global solution of equation (12) on $I \times \mathbb{S}^{2}$, then $m$ satisfies

$$
r \partial_{r} m-\beta \cdot \not \nabla m=r \Gamma \frac{\not \Delta u}{u}-(2 A-1) m+r(A-B) .
$$

We note that if $r|2 A-1| \leq C$, and

$$
|A-B|^{*} \in L^{1}(I),
$$

then using the maximum principle, it follows that $|m|$ is bounded. Applying Schauder theory to equation (22) we then obtain:

Theorem 4. Let $I=\left[r_{0}, \infty\right)$, let $\beta, \Gamma, A, B \in H_{I}^{2, \alpha}$, suppose that (23) is satisfied, and let $u \in H_{I}^{2, \alpha}$ be a positive solution of equation (12). Suppose that there is a constant $C>0$ such that

$$
\begin{gathered}
\|u\|_{2, \alpha ; I},\|r(2 A-1)\|_{2, \alpha ; I},\|B\|_{2, \alpha ; I},\left\||A-B|^{*}\right\|_{L^{1}(I)} \leq C, \\
C^{-1} \leq \Gamma \leq C .
\end{gathered}
$$

Then $m=r\left(1-u^{-2}\right) / 2$ satisfies

$$
\|m\|_{4, \alpha ; I}<C^{\prime}
$$

where $C^{\prime}$ depends only on $C$.

We will also need the bound (24) in some cases where the condition (23) cannot be verified. To this end, we state a comparison result that establishes a bound on $m$. Let $u_{i}, i=1,2$, satisfy

$$
r \partial_{r} u_{i}-\beta \cdot \not \triangleright u_{i}=\Gamma u_{i}^{2} \not \Delta u_{i}+A u_{i}-B_{i} u_{i}^{3} .
$$

Setting $m_{i}=r\left(1-u_{i}^{-2}\right) / 2, i=1,2$, and $\tilde{m}=m_{2}-m_{1}$, we obtain, in view of (22),

$$
r \partial_{r} \tilde{m}-\tilde{\beta} \cdot \not \mathbf{m}=\Gamma u_{2}^{2} \not \mathbf{\Delta} \tilde{m}+(2 \tilde{A}-1) \tilde{m}+r\left(B_{1}-B_{2}\right),
$$

where

$$
\begin{aligned}
& \tilde{\beta}=\beta+3 \Gamma u_{2}^{4} \not\left(m_{1}+m_{2}\right), \\
& \tilde{A}=A-\Gamma u_{1}^{2} u_{2}^{2}\left(r^{-1} \not \Delta m_{1}+\left.3 r^{-2}\left(u_{1}^{2}+u_{2}^{2}\right)|\not| m_{1}\right|^{2}\right) \text {. }
\end{aligned}
$$

If we also assume that $m_{1}, \not \supset m_{1}, \not \Delta m_{1}, u_{1}$, and $u_{2}$ are bounded, then $r|2 \tilde{A}-1|$ is bounded, and provided that also $\left|B_{1}-B_{2}\right|^{*} \in L^{1}(I)$, we may apply the previous argument to derive a bound on $|\tilde{m}|$, and consequently also on $\left|m_{2}\right|$. This leads to the following theorem.

Theorem 5. Let $I=\left[r_{0}, \infty\right)$, let $\beta, \Gamma, A, B_{1}, B_{2} \in H_{I}^{2, \alpha}$ and suppose that $u_{i}>0$, $i=1,2$, are bounded classical solutions of equation (25) on $I \times \mathbb{S}^{2}$. Let $B=B_{1}-B_{2}$, and suppose also that $|B|^{*} \in L^{1}(I)$, that $m_{1}$ is bounded, and that there is a constant $C>0$ such that

$$
\begin{gathered}
\left\|u_{1}\right\|_{2, \alpha ; I},\left\|u_{2}\right\|_{2, \alpha ; I},\left\|m_{1}\right\|_{2, \alpha ; I},\|r(2 A-1)\|_{2, \alpha ; I},\|B\|_{2, \alpha ; I},\left\||B|^{*}\right\|_{L^{1}(I)} \leq C, \\
C^{-1} \leq \Gamma \leq C .
\end{gathered}
$$


Then $\tilde{m}=r\left(1-\tilde{u}^{-2}\right) / 2$ satisfies

$$
\|\tilde{m}\|_{4, \alpha ; I} \leq C^{\prime}
$$

where $C^{\prime}$ depends only on $C$.

\subsection{Continuous dependence on parameters.}

Theorem 6. Let $I=\left[r_{0}, \infty\right)$, and suppose that $u_{\lambda} \in H_{I}^{k+2, \alpha}, a \leq \lambda \leq b$, is a family of solutions of (12) with $\beta_{\lambda}, \Gamma_{\lambda}, A_{\lambda}, B_{\lambda}$ satisfying $r\left(2 A_{\lambda}-1\right), r\left(2 B_{\lambda}-1\right), \beta_{\lambda}, \Gamma_{\lambda} \in$ $C^{0}\left([a, b], H_{I}^{k, \alpha}\right)$ and with the initial data $u_{\lambda}\left(r_{0}\right) \in C^{0}\left([a, b], C^{k+2, \alpha}\left(\mathbb{S}^{2}\right)\right)$. Suppose also that one of the following conditions is satisfied:

1. $\left|A_{\lambda}-B_{\lambda}\right|^{*} \in C^{0}\left([a, b], L^{1}(I)\right)$;

2. A continuous family of solutions $m_{\lambda}^{\prime} \in C^{0}\left([a, b], H_{I}^{k+2, \alpha}\right)$ of (22) exist.

Then $u_{\lambda}, m_{\lambda} \in C^{0}\left([a, b], H_{I}^{k, \alpha}\right)$.

\section{Deformation of metrics in $\mathcal{M}$}

We are now in a position to sketch the proof of the Main Theorem. Recall that any metric $g \in \mathcal{M}$ can be written as in (7). We define a nested sequence of subsets $\mathcal{M}=\mathcal{M}_{0} \supset \cdots \supset \mathcal{M}_{4}$ :

$\mathcal{M}_{1}=\left\{g \in \mathcal{M}_{0}: r(1-u) \in H_{\left[r_{0}, \infty\right)}^{4, \alpha} ; r \beta, r v \in H_{\left[r_{0}, \infty\right)}^{8, \alpha}, \forall r_{0}>0, \operatorname{supp} R\right.$ is compact $\}$, $\mathcal{M}_{2}=\left\{g \in \mathcal{M}_{1}: 2 \kappa-R>0\right\}$,

$\mathcal{M}_{3}=\left\{g \in \mathcal{M}_{2}: \beta, v\right.$ are compactly supported $\}$,

$\mathcal{M}_{4}=\left\{g \in \mathcal{M}_{3}: g\right.$ is flat $\}$.

Let us say that $\mathcal{M}_{i}$ is connected to $\mathcal{M}_{i+1}$ if for each $g \in \mathcal{M}_{i}$ there is a path $\Gamma$ in $\mathcal{M}_{i}$, continuous in the topology of $C_{-1}^{2, \alpha}$, with $\Gamma(0)=g$ and $\Gamma(1) \in \mathcal{M}_{i+1}$. We will show that $\mathcal{M}_{i}$ is connected to $\mathcal{M}_{i+1}$ for each $i=0, \ldots, 3$. The Main Theorem follows by joining these paths.

Lemma 7. $\mathcal{M}_{0}$ is connected to $\mathcal{M}_{1}$.

Proof. Let $g=g_{0} \in \mathcal{M}_{0}$. It is not difficult, using a truncation followed by a standard smoothing, to construct a deformation $g_{\lambda}$, continuous in $C_{-1}^{2, \alpha}$, from $g_{0}$ to a smooth metric $g_{1}$ which is flat outside a large enough ball, with scalar curvature $R_{\lambda} \in L^{1}$, and such that $g_{\lambda}-g_{0}$ is small in $C_{-1 / 2}^{2, \alpha}$ for all $\lambda$. Since $g_{\lambda}$ is close to $g$, the coordinate spheres are still quasiconvex in $g_{\lambda}$, and the negative part $R_{\lambda}^{-}$of the scalar curvature of $g_{\lambda}$ is small in $L^{3 / 2}$. It follows that the operator $-8 \Delta_{g_{\lambda}}+R_{\lambda}$ is injective [4], and hence also a bijection; see [5, 12]. We can now choose a smooth positive function of compact support $S_{\lambda}$ which is close to $R_{\lambda}$ in $C_{-5 / 2}^{\alpha}$, and solve the equation

$$
\left(-8 \Delta+R_{\lambda}\right) \psi_{\lambda}=R_{\lambda}-S_{\lambda}
$$

It follows from the above that $\psi_{\lambda}$ is small in $C_{-1 / 2}^{2, \alpha}$. Taking $\phi_{\lambda}=1+\psi_{\lambda}$, we see that the metrics $\phi_{\lambda}^{4} g_{\lambda}$ have positive scalar curvature, quasiconvex coordinate spheres, and form a continuous path from $g_{0}$ to a smooth metric $\tilde{g}_{1}=\phi_{1}^{4} g_{1}$. Since $R_{1}-S_{1}$ is of compact support, and $g_{1}$ is flat outside a compact set, it follows that $\tilde{g}_{1} \in C_{-1}^{k, \alpha}$ for all $k$. On each coordinate sphere equipped with the metric $\gamma$ induced by $\tilde{g}_{1}$, it is possible, using the techniques of [8, Chapter 2], to find a uniformization factor $r^{2} e^{2 v}$, with bounds as required in $\mathcal{M}_{1}$, so that $\bar{\gamma}=r^{-2} e^{-2 v} \gamma$ is a round metric 
with surface area $4 \pi r^{2}$. We conclude that the continuous path $\phi_{\lambda}^{4} g_{\lambda}$ joins $g \in \mathcal{M}_{0}$ to a metric $\tilde{g}_{1} \in \mathcal{M}_{1}$.

It is important to note that since the round metric $\bar{\gamma}$ on the coordinate spheres will in general vary with $r$, it is most likely necessary to change the background flat metric when writing $\tilde{g}_{1}$ as in (7). Nevertheless, these two flat metrics are asymptotic as $r \rightarrow \infty$; see 13 for details.

As outlined in the Introduction, the deformation is obtained in the next three steps by deforming $g_{\lambda}$ explicitly inside a ball $B_{r_{0}}$, while solving (12) outside $B_{r_{0}}$ with the deformation of $\beta_{\lambda}, v_{\lambda}$ and $R_{\lambda}$ defined so that $\kappa_{\lambda}, \bar{H}_{\lambda}>0, R_{\lambda} \geq 0$, and so that theorems from Section 2 guarantee global existence, asymptotic behavior as $r \rightarrow \infty$, and continuity of $u_{\lambda}$ in $H_{\left[r_{0}, \infty\right)}^{4, \alpha}$. Note that in order to ensure continuity at the end point of the deformation, it is necessary to have a $\bar{H}_{\lambda}$ uniformly bounded below by a positive constant. Now, if $g_{\lambda}$ is a path in $\mathcal{M}_{i}, i=1,2,3$, such that for some $0<r^{\prime}<r_{0},\left.g_{\lambda}\right|_{B_{r_{0}}}$ is continuous in $C^{2}$, and $r\left(1-u_{\lambda}\right), r \beta_{\lambda}$, and $r v_{\lambda}$ are continuous in $H_{\left[r^{\prime}, \infty\right)}^{4, \alpha}$, then $g_{\lambda}$ is continuous in $C_{-1}^{2, \alpha}$.

Lemma 8. $\mathcal{M}_{1}$ is connected to $\mathcal{M}_{2}$.

Proof. Let $g \in \mathcal{M}_{1}$, choose $r_{1}$ such that $2 \kappa-R>0$ for $r<r_{1}$, and $r_{0}$ with $r_{0}<r_{1}$. For each $\lambda \in[0,1]$, define $g_{\lambda}=g$ inside $B_{r_{0}}, \beta_{\lambda}=\beta$, and $v_{\lambda}=v$ everywhere. We have $\kappa_{\lambda}, \bar{H}_{\lambda}>0$. Let $\varphi(r)$ be a smooth cut-off function on $[0, \infty)$, satisfying $0 \leq \varphi \leq 1, \varphi=1$ on $\left[0, r_{0}\right]$, and $\varphi=0$ on $\left[r_{1}, \infty\right)$. Define $\varphi_{\lambda}(r)=(1-\lambda)+\lambda \varphi(r)$ and define $R_{\lambda}=\varphi_{\lambda} R$. Then $R_{\lambda}$ is monotonically decreasing in $\lambda, R_{\lambda}=R$ on $B_{r_{0}}$, and $\operatorname{supp}\left(R_{1}\right) \subset B_{r_{1}}$. Thus, Theorems 3 and 5 can be used to solve equation (12) on $\left[r_{0}, \infty\right) \times \mathbb{S}^{2}$ for $u_{\lambda} \in H^{4, \alpha}$. The continuity of $u_{\lambda}$ and $m_{\lambda}$ with respect to $\lambda$ is obtained from Theorem 6. Clearly, $g_{1} \in \mathcal{M}_{2}$ and the lemma follows.

Lemma 9. $\mathcal{M}_{2}$ is connected to $\mathcal{M}_{3}$.

Proof. Let $g \in \mathcal{M}_{2}$, and put $R_{\lambda}=R$. For $\lambda \in[1, \infty)$ define $\tilde{\beta}_{\lambda}=\left(\phi_{\lambda}\right)^{*} \beta, \tilde{v}_{\lambda}=$ $\left(\phi_{\lambda}\right)^{*} v$, where $\phi_{\lambda}(r, \theta)=(\lambda r, \theta)$. Note that $r \tilde{\beta}_{\lambda}$ and $r \tilde{v}_{\lambda}$ are continuous in $H_{I}^{6, \alpha}$ since $r \tilde{\beta}_{\lambda}$ and $r \tilde{v}_{\lambda}$ are uniformly bounded in $H_{I}^{8, \alpha}$. Now, let $\beta_{\lambda}=\varphi \beta+(1-\varphi) \tilde{\beta}_{\lambda}$, and define $v_{\lambda}$ by $e^{2 v_{\lambda}}=\varphi e^{2 v}+(1-\varphi) e^{2 \tilde{v}_{\lambda}}$, where $\varphi(r)$ is a cut-off function as in the proof of the previous lemma. It follows from (9) that

$$
e^{2 v_{\lambda}} \bar{H}_{\lambda}=\varphi e^{2 v} \bar{H}+(1-\varphi) e^{2 \tilde{v}_{\lambda}} \tilde{H}_{\lambda}+\left(e^{2 v}-e^{2 \tilde{v}_{\lambda}}\right) r \varphi^{\prime},
$$

where $\tilde{H}_{\lambda}=\left(\phi_{\lambda}\right)^{*} \bar{H}$. Thus, since $v$ and $\tilde{v}_{\lambda}$ tend to zero as $r \rightarrow \infty$, it follows that if $r_{0}$ and $r_{1} / r_{0}$ are large enough, then $\bar{H}_{\lambda}>0$ for $r>r_{0}$. Furthermore, in view of (10), the Gauss curvature $\kappa_{\lambda}$ is given by

$$
\begin{aligned}
r^{2} e^{2 v_{\lambda}} \kappa_{\lambda}=1-\not \Delta v_{\lambda}=r^{2}\left(\varphi e^{2 v} \kappa\right. & \left.+(1-\varphi) e^{2 \tilde{v}_{\lambda}} \tilde{\kappa}_{\lambda}\right)+|\not \downarrow v|^{2} \\
& -2 e^{-2 v_{\lambda}}\left(\left.\varphi e^{2 v}|\not| v\right|^{2}+\left.(1-\varphi) e^{2 \tilde{v}_{\lambda}}|\not| \tilde{v}_{\lambda}\right|^{2}\right),
\end{aligned}
$$

where $\tilde{\kappa}_{\lambda}=\left(\phi_{\lambda}\right)^{*} \kappa$. Hence, since also $|\not \downarrow v|$ and $|\not| \tilde{v}_{\lambda} \mid$ tend to zero as $r \rightarrow \infty$, we see that if $r_{0}$ is large enough, then $\kappa_{\lambda}>0$ for $r>r_{0}$. By choosing $r_{0}$ large enough, we can also ensure that $R=0$ outside $B_{r_{0}}$. As in the proof of the previous lemma, we define $g_{\lambda}=g$ inside $B_{r_{0}}$, and solve equation (12) for $u_{\lambda}$ outside $B_{r_{0}}$. The existence of $u_{\lambda}$ for all $r \geq r_{0}$ is now guaranteed by Theorem 1. Note that outside $B_{r_{1}}, \beta_{\lambda}=\tilde{\beta}_{\lambda}, v_{\lambda}=\tilde{v}_{\lambda}$, hence we have a uniformly bounded solution $\lambda^{-1}\left(\phi_{\lambda}\right)^{*} m$ of equation (22), and therefore Theorem [5]applies to give the asymptotic behavior 
of $u_{\lambda}$ for $r \rightarrow \infty$. It is easy to see that the path $g_{\lambda}$ can be extended continuously to $[1, \infty]$, and since $\beta_{\lambda}$ and $v_{\lambda}$ tend to zero as $\lambda \rightarrow \infty$ for $r>r_{1}$, it follows that $g_{\infty} \in \mathcal{M}_{3}$.

Lemma 10. $\mathcal{M}_{3}$ is connected to $\mathcal{M}_{4}$.

Proof. Let $g \in \mathcal{M}_{3}$, choose $r_{0}>0$ so that $R, \beta$, and $v$ are supported in $B_{r_{0}}$, and let $\varphi(r)$ be a cut-off function as above. For $\lambda \in[1, \infty)$, define $\beta_{\lambda}=\varphi\left(\phi_{1 / \lambda}\right)^{*} \beta$, and $\tilde{v}_{\lambda}=\left(\phi_{1 / \lambda}\right)^{*} v$. Let $r_{0}<r_{1}<r_{2}$, and let $\zeta(r)$ be a smooth function satisfying $0 \leq \zeta \leq 1, \zeta=1$ on $\left[0, r_{1}\right], \zeta=0$ on $\left[r_{2}, \infty\right)$. Let $\tilde{H}_{\lambda}=\left(\phi_{1 / \lambda}\right)^{*} \bar{H}$, and note that $\inf \tilde{H}_{\lambda}=\inf \bar{H}>0$, hence $h=\inf \tilde{H}_{\lambda}$ is independent of $\lambda$. Let $f(r)$ be a smooth non-negative function supported on $\left[r_{0}, r_{2}\right]$, satisfying on $\left[r_{0}, r_{1}\right]$ the inequality:

$$
f>-r^{-a-1}\left(\varphi h+r \varphi^{\prime}\right)
$$

where $a=\max \left\{-\left(2+2 r \partial_{r} \tilde{v}_{\lambda}\right), 0\right\}$. Let $\xi(r)=r^{a} \int_{r_{0}}^{r} f(s) d s, \psi=\xi+\varphi$, and $v_{\lambda}=\zeta\left(\tilde{v}_{\lambda}+\frac{1}{2} \log \psi\right)$. Since $\xi \geq 0$, it now follows from (9) that we have for $r_{0}<r<r_{1}$ :

$$
\psi \bar{H}_{\lambda}=\xi\left(2+2 r \partial_{r} \tilde{v}_{\lambda}\right)+r \xi^{\prime}+\varphi \tilde{H}_{\lambda}+r \varphi^{\prime}>-a \xi+r \xi^{\prime}-r^{a+1} f=0 .
$$

Furthermore, since $\beta_{\lambda}=0$ in $B_{r_{2}} \backslash B_{r_{1}}$, we can also choose $\zeta$ so as to ensure that $\bar{H}_{\lambda}>0$ there, provided that $r_{2} / r_{1}$ is large enough. Since the deformation of $v$ is radial, it is clear that $\kappa_{\lambda}>0$. Define $g_{\lambda}=\lambda^{2}\left(\phi_{1 / \lambda}\right)^{*} g$ in $B_{r_{0}}$, and as before, solve for $u_{\lambda}$ in (12) on $\left[r_{0}, \infty\right) \times \mathbb{S}^{2}$ with initial data $\left.u_{\lambda}\right|_{S_{r_{0}}}$. Global existence and asymptotic behavior as $r \rightarrow \infty$ is obtained from Theorems 1 and 4 . The path $g_{\lambda}$ can be extended continuously to $[1, \infty]$, and since $\beta_{\lambda}, v_{\lambda}$, and $R_{\lambda}$ tend to zero as $\lambda \rightarrow \infty$, it follows that $u_{\lambda}$ tends to 1 . Consequently $g_{\infty}$ is flat, and the continuous path $g_{\lambda}$ joins $g_{1}$ to a flat metric $g_{\infty} \in \mathcal{M}_{4}$. However, note that $g_{1} \neq g$, since clearly $v_{1} \neq v$. In order to complete the proof of the lemma, we now define a continuous path $g_{\lambda}, \lambda \in[0,1]$, between $g$ and $g_{1}$. Define $g_{\lambda}=g$ in $B_{r_{0}}$, $\beta_{\lambda}=\beta, R_{\lambda}=R$, and $v_{\lambda}=\zeta(v+(1 / 2) \log (1-\lambda+\lambda \psi))$. Then from (9) we get $e^{2 v_{\lambda}} \bar{H}_{\lambda}=(1-\lambda) e^{2 v_{0}} \bar{H}_{0}+\lambda e^{2 v_{1}} \bar{H}_{1}>0$ in $\left[r_{0}, r_{1}\right]$, and as before $\bar{H}_{\lambda}>0$ also in $\left[r_{1}, r_{2}\right]$ provided $r_{2} / r_{1}$ is large enough. Clearly, as before, we have $\kappa_{\lambda}>0$. Thus, we can solve for $u_{\lambda}$ in (12) as above.

Note that due to the uniformization step in Lemma 7 , the final flat metric $g_{\infty}$ in Lemma 10 may be different from the background flat metric originally given in $\mathcal{M}_{0}$.

\section{REFERENCES}

1. R. Bartnik, Existence of maximal surfaces in asymptotically flat spacetimes, Comm. Math. Phys. 94 (1984), no. 2, 155-175. MR 86b:53063

2. R. Bartnik, Quasispherical metrics and prescribed scalar curvature, J. Differential Geom. 37 (1993), no. 1, 31-71. MR 93i:53041

3. M. Cantor, A necessary and sufficient condition for York data to specify an asymptotically flat spacetime, J. Math. Phys. 20 1979, 1741-1744. MR 80k:83018

4. M. Cantor and D. Brill, The Laplacian on asymptotically flat manifolds and the specification of scalar curvature, Comp. Math. 43 (1981), fasc. 3, 317-330. MR 83a:58021

5. A. Chaljub-Simon and Y. Choquet-Bruhat, Problèmes elliptiques du second ordre sur une variété euclidienne à l'infini, Ann. Fac. Sci. Toulouse Math. 5 (1979), no. 1, 9-25. MR 80i:58044

6. Y. Choquet-Bruhat, Global solutions of the constraints equations on open and closed manifolds, GRG 5 (1974), no. 1, 49-60. MR 53:10100 
7. Y. Choquet-Bruhat, J. Isenberg, and J. W. York, Jr, Einstein constraints on asymptotically Euclidean manifolds, Phys. Rev. D. 61 (2000), article \# 084034.

8. D. Christodoulou and S. Klainerman, The global nonlinear stabililty of the Minkowski space, Princeton Mathematical Series, No. 41, Princeton University Press, Princeton, 1993. MR 95k:83006

9. D. Christodoulou and N. O'Murchadha, The boost problem in general relativity, Comm. in Math. Phys. 80 (1981), no. 2, 271-300. MR 84e:83011

10. A. E. Fischer, J. E. Marsden, and V. Moncrief, The structure of the space of solutions of Einstein's equations. I, One Killing field, Ann. Inst. H. Poincaré Sect. A (N.S.), 33 (1980), no. 2, 147-194. MR 82d:83007

11. O. A. Ladyženskaja, V. A. Solonnikov, and N. N. Ural'ceva, Linear and quasilinear equations of parabolic type, Transl. of Math. Mono., Vol. 23, AMS, Providence, RI, 1968. MR 39:3159b

12. J. M. Lee and T. H. Parker, The Yamabe problem, Bull. Amer. Math. Soc. (N.S.) 17 (1987), no. 1, 37-91. MR 88f:53001

13. B. Smith and G. Weinstein, Quasiconvex foliations and asymptotically flat metrics of nonnegative scalar curvature, in preparation.

14. R. M. Wald, General relativity, The University of Chicago Press, Chicago, IL, 1984. MR 86a:83001

15. J. W. York, Jr., Gravitational degrees of freedom and the initial-value problem, Phys. Rev. Lett. 23 (1971), no. 26, 1656-1658. MR 44:7944

University of Alabama at Birmingham, Birmingham, AL 35205

E-mail address: smith@math.uab.edu

University of Alabama at Birmingham, Birmingham, AL 35205

E-mail address: weinstei@math.uab.edu 